LAWRENCE LIVERMORE N A TION AL LABORATORY

\title{
Xradia Performance Summary - Update
}

A. M. Waters, H. E. Martz, D. J. Chinn, C. M.

Logan, J. C. Gross, D. Scott

September 15, 2004 
This document was prepared as an account of work sponsored by an agency of the United States Government. Neither the United States Government nor the University of California nor any of their employees, makes any warranty, express or implied, or assumes any legal liability or responsibility for the accuracy, completeness, or usefulness of any information, apparatus, product, or process disclosed, or represents that its use would not infringe privately owned rights. Reference herein to any specific commercial product, process, or service by trade name, trademark, manufacturer, or otherwise, does not necessarily constitute or imply its endorsement, recommendation, or favoring by the United States Government or the University of California. The views and opinions of authors expressed herein do not necessarily state or reflect those of the United States Government or the University of California, and shall not be used for advertising or product endorsement purposes.

This work was performed under the auspices of the U.S. Department of Energy by University of California, Lawrence Livermore National Laboratory under Contract W-7405-Eng-48. 


\title{
Xradia Performance Summary - Update ${ }^{1}$
}

\author{
Amy Waters, Harry Martz, Diane Chinn, Clint Logan, Jeff Gross and David Scott ${ }^{*}$ \\ Lawrence Livermore National Laboratory, Livermore, CA \\ "Xradia, Inc., Concord, CA
}

\section{Introduction}

High Energy Density Physics (HEDP) Experiments play an important role in corroborating the improved physics codes that underlie LLNL's Stockpile Stewardship mission. Conducting these experiments, whether on the National Ignition Facility (NIF) or another national facility such as Omega, will require not only improvement in the diagnostics for measuring the experiment, but also detailed knowledge of the as-built target components and assemblies themselves. To assist in this effort, a defined set of well-known reference standards designed to represent a range of HEDP targets have been built and are being used to quantify the performance of different characterization techniques. Without the critical step of using reference standards for qualifying characterization tools there can be no verification of either commercial or internallydeveloped characterization techniques and thus an uncertainty in the as-built-model for the initial condition to the physics codes.

In FY03, two reference standards were fabricated and characterized using metrology tools. One of the reference standards was built with a cylindrical geometry and contained features similar to those on a Super Nova Raleigh Taylor (SNRT) target. The other reference standard was built with a spherical geometry and contained features similar to those on a Double Shell target. The standards were designed for manufacturability, stability and to provide a range of features that can be measured using NDE methods [Hibbard, et al. 2004]. A tantalum edge was used to determine the digital radiographic modulation transfer function.

\section{Xradia $\mu$ XCT system}

Xradia's $\mu \mathrm{XCT}$ system is a high-resolution digital radiography (DR) and computed tomography (CT) system comprised of an X-ray source, detector, and a sample translation stage capable of $x, y, z$ and theta rotation. The source is a $160-\mathrm{kVp}$ microfocus $\mathrm{X}$-ray tube with a spot size of $5 \mu \mathrm{m}$, and a tungsten target. The detector is a scintillator with a microscope lens coupled to a $1024 \times 1024$ TE cooled CCD camera with a 16-bit A/D converter. The location of the detector is easily moveable with respect to the source,

\footnotetext{
${ }^{1}$ This work was performed under the auspices of the U. S. Department of Energy by the University of California Lawrence Livermore National Laboratory under contract No. W-7405-Eng-48.
} 
which results in a change in magnification, and hence pixel size at the object and spatial resolution. DR and CT data can be acquired at any detector location and magnification. For this project DR images were acquired at both high- and low-resolution detector locations, however CT data was only acquired in low-resolution modes due to the limited field-of-view for the high-spatial resolution modes of operation. For results presented here, the source-to-object distance was approximately $100 \mathrm{~mm}$, and the object-to-detector distance was approximately $50 \mathrm{~mm}$ for both reference standards. The different detector locations and resulting high- and low-resolution imaging modes used for each reference standard are summarized in Table 1.

Table 1. Radiographic and CT spatial resolution modes utilized with the Xradia $\mu$ XCT system.

\begin{tabular}{|c|c|c|}
\hline \multirow{2}{*}{ Reference Standard } & \multicolumn{2}{|c|}{ Pixel size at object $(\boldsymbol{\mu} \mathbf{m})$} \\
\cline { 2 - 3 } & Low-resolution mode & High-resolution mode \\
& DR and CT & DR \\
\hline Spherical & 1.45 & 0.47 \\
\hline Cylindrical & 2.31 & 0.5 \\
\hline
\end{tabular}

\section{Digital radiography system performance}

In an attempt to begin characterizing the digital radiography system performance with respect to the reference standards, we have acquired data nondestructively using several different $\mathrm{x}$-ray DR and CT systems. Reports are being written to determine the performance of each DR/CT system investigated and used to characterize these standards; for example, for LLNL's KCAT performance see Waters, et al. 2004. Here we present the report on Xradia's $\mu$ XCT system.

Digital radiographic and tomographic data were acquired with the $\mu \mathrm{XCT}$ system. For acquiring DR images and CT data, $60-\mathrm{kV}$ electron excitation voltage was used with a current of around $0.1 \mathrm{~mA}$. The optimum energy appropriate for DR and CT of these reference standards was determined to be $\sim 8 \mathrm{keV}$ [Logan, et al. 2001]. Digital radiographs were acquired for both standards using higher resolution modes that yielded a pixel size at the object of $0.466 \mu \mathrm{m}$, with a total field of view of $477 \mu \mathrm{m} \times 477 \mu \mathrm{m}$ for the spherical standard, and a pixel size of $0.5 \mu \mathrm{m}$, with a total field of view of $512 \mu \mathrm{m} \mathrm{x}$ $512 \mu \mathrm{m}$ for the cylindrical standard.

Several different methods have been employed to determine the spatial and contrast performance of DR systems. Here we describe a few techniques that we use. In order to begin quantifying digital radiography system performance, a polished thin $(0.51 \mathrm{~mm})$ tantalum edge was imaged using identical DR/CT data acquisition parameters as used for the reference standards. Percent transmission images $\left[\left(I / \mathrm{I}_{0}\right) \times 100\right]$ were created and 10pixel wide one-dimensional lineouts were taken from the polished edge to determine the edge response of the system. Two lineouts taken from different locations across the tantalum edge image are shown in Figure 1. 


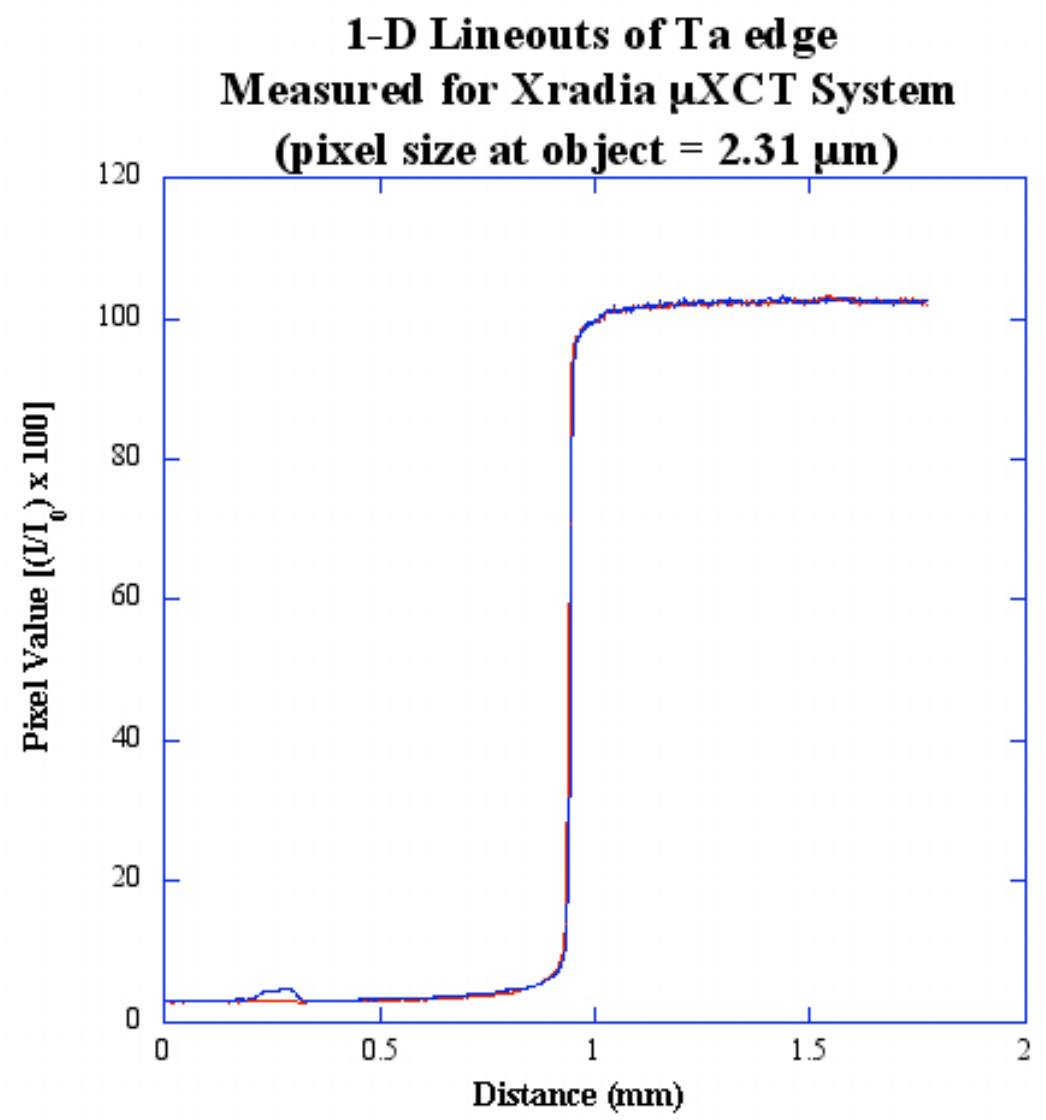

Figure 1. Two 10-pixel wide one-dimensional lineouts were taken across the thin tantalum edge transmission image. Two lineouts were used to measure MTF to demonstrate repeatability of this technique.

The lineouts shown in Figure 1 were taken in the 2.31- $\mu \mathrm{m}$ imaging mode that was used to acquire DR and CT data for the cylindrical standard. The one-dimensional lineouts from the image of the Ta edge were used to calculate the projection or DR Modulation Transfer Function (MTF) of the system. To calculate the MTF for the Xradia $\mu$ XCT system, the derivative of the lineout (edge response) was calculated, resulting in what is called the line-spread function. The Fourier transform of the line-spread function is the MTF. The MTF is a frequency-domain description of the spatial resolution of an imaging system or component [Hasegawa, et al. 1991; Logan, et al. 1998]. The MTF of a system is the product of the MTFs of each of the components individually, and thus is a preferred technique for many imaging experts. The MTF of a system is usually presented as a graph with frequency in $\mathrm{mm}^{-1}$, or linepairs per millimeter $(\mathrm{lp} / \mathrm{mm})$ on the horizontal, or X-axis. At low-spatial frequencies, the MTF usually approaches 1 . The MTF falls with increasing frequency, and can never exceed a sinc function $[\sin (\mathrm{x}) / \mathrm{x}]$, where $\mathrm{x}$ is the pixel size. Modulation as a function of frequency in $1 \mathrm{p} / \mathrm{mm}$ is presented in Figure 2 for the Xradia $\mu$ XCT system. 


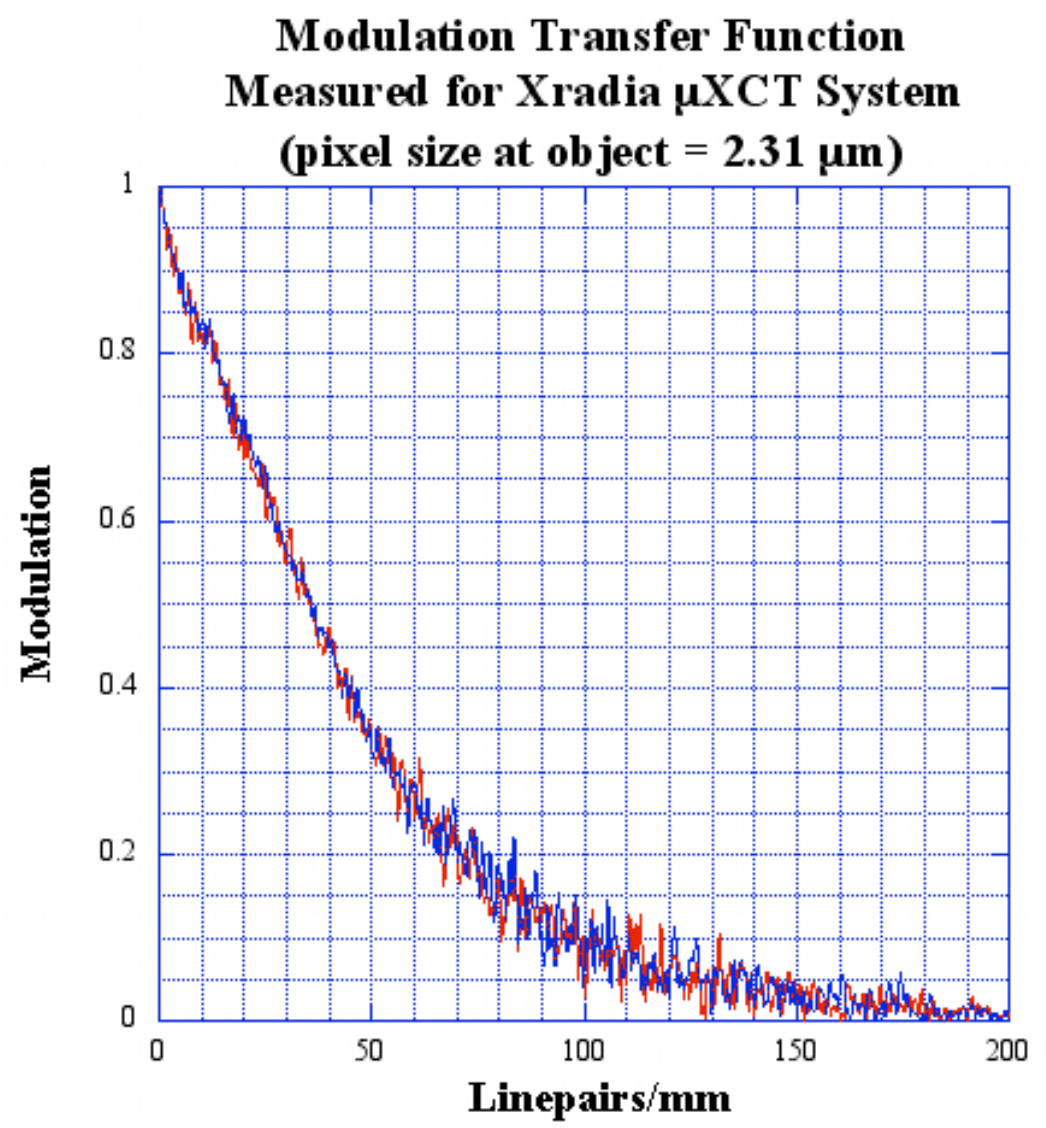

Figure 2. Two MTFs measured for the Xradia $\mu$ XCT system using the two lineouts shown in Figure 1. At $20 \mathrm{lp} / \mathrm{mm}$, the modulation is $\sim 70 \%$.

In addition to the edge images used to measure the MTF of the system, Xradia also provided us with images of the tantalum edge that had been deconvolved with a point spread function (psf) assumed to represent the Xradia source. These images provided a qualitatively "sharper" image. The lineouts from one deconvolved and non-deconvolved edge image are shown in Figure 3, below. It is useful to note the under and overshoot across the edge in the deconvolved image lineout as compared to the lineout from the raw or non-deconvolved image. The resulting MTF measurement using the lineout from the deconvolved image gives results that are physically unrealistic, such as values greater than 1. Hence, all MTF measurements presented in this report were performed on the non-deconvolved edge images. 


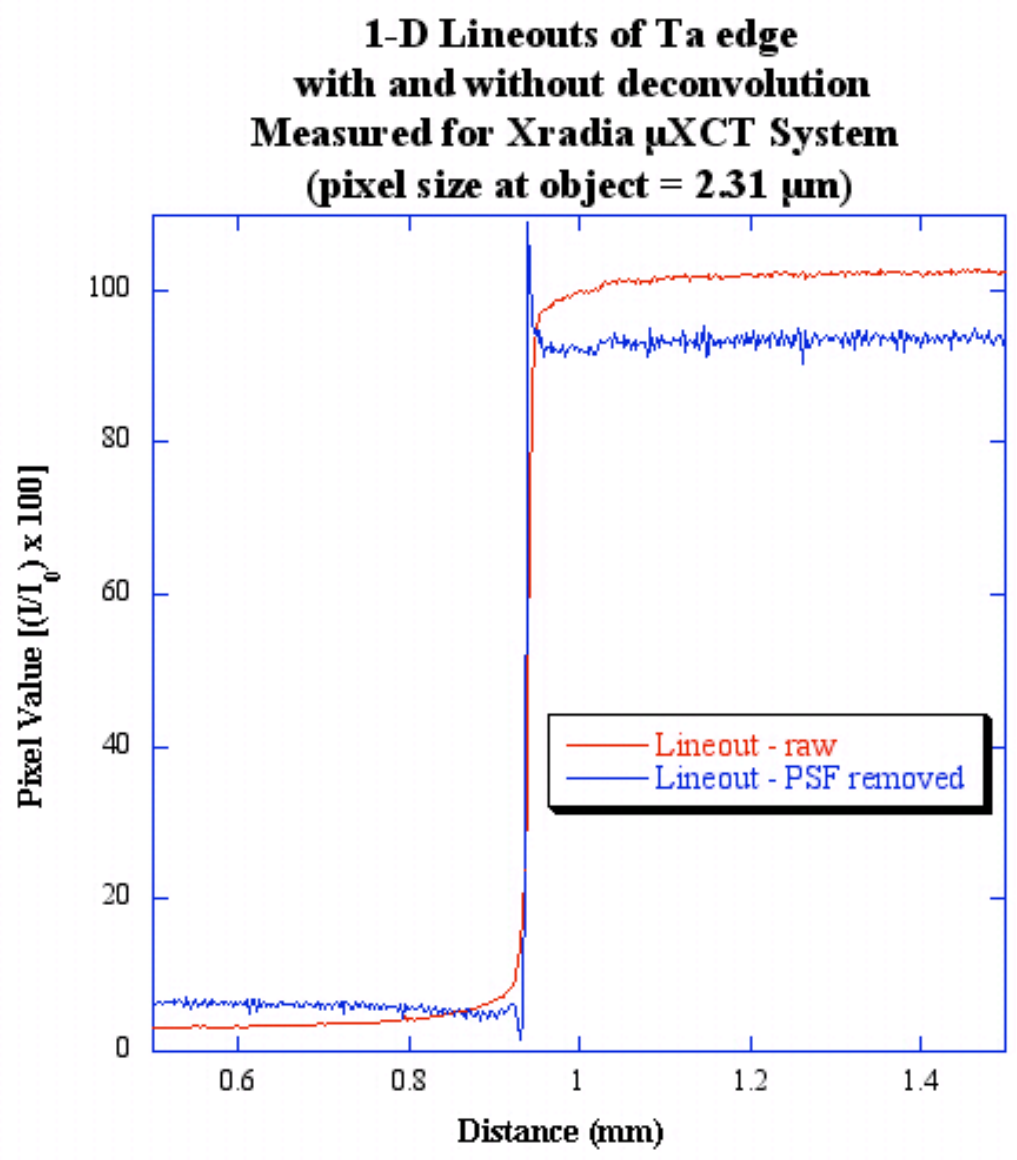

Figure 3. Two 1-D lineouts taken of the Ta-edge image, with and without the deconvolution of the assumed point spread function. Note the overshoot of the blue line at both edges.

The lineouts acquired from the Ta-edge images in the higher resolution modes (pixel size $=0.47$ and $0.5 \mu \mathrm{m}$ ) show effects possibly due to phase contrast that result in an overshoot near the edge, as shown in Figure 4. This overshoot in the edge step functions again gave physically unrealistic MTF results such as values greater than 1. Thus MTF measurements are not presented for the higher resolution imaging modes. 


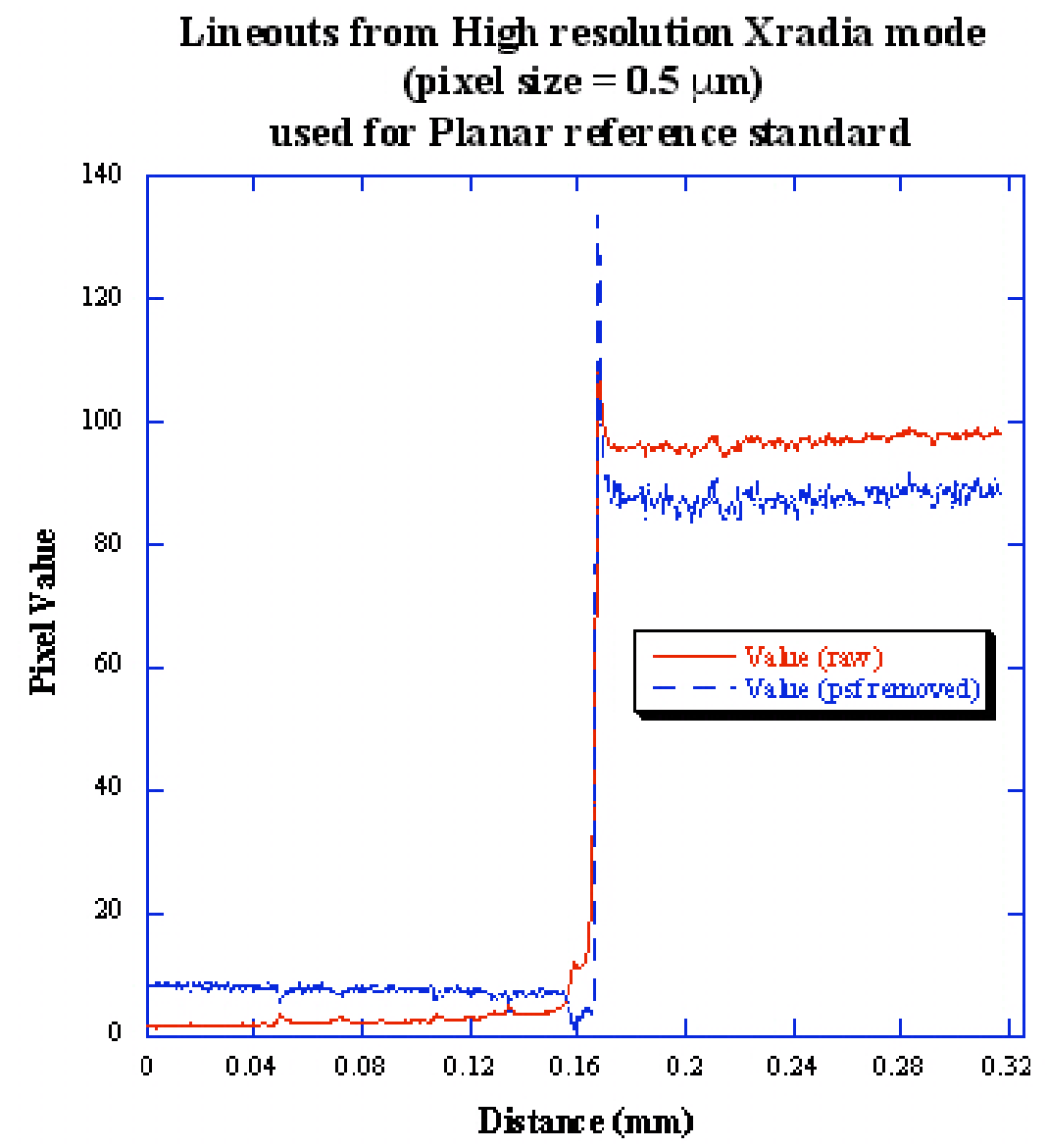

Figure 4. Two 1-D lineouts taken of the Ta-edge image in the high-resolution mode, with and without the deconvolution of an assumed point spread function. Note the overshoot of the red and the blue line at the edge.

A rule of thumb method used to determine spatial resolution is to multiply the pixel size at the object by a factor of 2.5. Using this quick calculation, for Xradia the resulting spatial resolution for the spherical reference standard is approximately $3.6 \mu \mathrm{m}$ in the CT acquisition mode and $1.2 \mu \mathrm{m}$ for the high resolution imaging mode. For the cylindrical reference standard, resulting spatial resolution for the low-spatial resolution mode is 5.8 $\mu \mathrm{m}$, and $1.3 \mu \mathrm{m}$ in the high-resolution mode.

Another quick and easy method to determine the spatial resolution of a system is to fit the line-spread function (calculated as the derivative of a one-dimensional lineout from an edge) with a Gaussian function. The resulting Full Width Half Maximum (FWHM) of the Gaussian can be multiplied by the pixel size at the object to give an indication of "worst-case" system resolution. The FWHM values and resulting spatial resolution as well as the rule of thumb spatial resolution results are summarized in Table 2. 
Table 2. Summary of estimated spatial resolution for different imaging modes using the FHWM and rule of thumb methods as described in the text.

\begin{tabular}{|c|c|c|c|c|}
\hline $\begin{array}{c}\text { Reference } \\
\text { Standard }\end{array}$ & $\begin{array}{c}\text { Imaging mode } \\
(\mu \mathrm{m})\end{array}$ & FWHM & $\begin{array}{c}\text { Spatial Res. } \\
\text { FWHM } \\
\text { technique }(\mu \mathrm{m})\end{array}$ & $\begin{array}{c}\text { Spatial Res. } \\
2.5 \mathrm{X} \text { technique } \\
(\mu \mathrm{m})\end{array}$ \\
\hline \multirow{2}{*}{ Spherical } & 1.45 & $\mathrm{~N}^{*} \mathrm{~A}^{*}$ & $\mathrm{~N}^{*}$ & 3.6 \\
\cline { 2 - 5 } Cylindrical & 0.47 & 4.8 & 2.3 & 1.2 \\
\cline { 2 - 5 } & 2.31 & 3.7 & 8.5 & 5.8 \\
\hline \multirow{2}{*}{ Cyyyy}
\end{tabular}

${ }^{*}$ We were not able to measure the FWHM for this data since it appears that all of the $1.45-\mu$ m mode Taedge images seem to be deconvolved with a psf.

Other techniques exist to quantify system performance, such as the Signal to Noise Ratio (SNR). The SNR is defined as the difference between the mean of two signals (in our case the two signals are in the Ta edge, and outside the Ta edge) divided by the squareroot of the sum of the squares of the standard deviation:

$$
S N R=\frac{S_{1}-S_{2}}{\sqrt{\sigma_{1}^{2}+\sigma_{2}^{2}}},
$$

where $S$ is the mean of the signal and $\sigma$ is the standard deviation of the signal. The SNR of the DR image of the tantalum edge can be calculated using the onedimensional lineouts taken from the tantalum edge transmission image, and can also be calculated over an area (two-dimensions). For the SNR results presented here $S_{l}$ is defined as the mean within the Ta-edge and $S_{2}$ is defined as the mean far from the edge. For the $\mu \mathrm{XCT}$ system, $2.31 \mu \mathrm{m}$ imaging mode, the two-dimensional DR SNR of the Taedge was determined for an area of 163 x 141 pixels, and was calculated to be 76 .

\section{Digital radiography of reference standards}

Xradia took several two-dimensional projection images of the reference standards in addition to acquiring CT data sets. These images were corrected for camera dark current and background, and are presented in Figure 5 below as percent transmission images $\left[\left(\mathrm{I} / \mathrm{I}_{0}\right) \mathrm{x} 100\right]$. As mentioned before, $\mathrm{x}$-ray attenuation is a function of $\mathrm{x}$-ray energy, path length, material density and elemental composition. In the images as shown below, dark regions indicate either longer path lengths, or higher density of material. Conversely, lighter regions then indicate less density of material or shorter path lengths. 


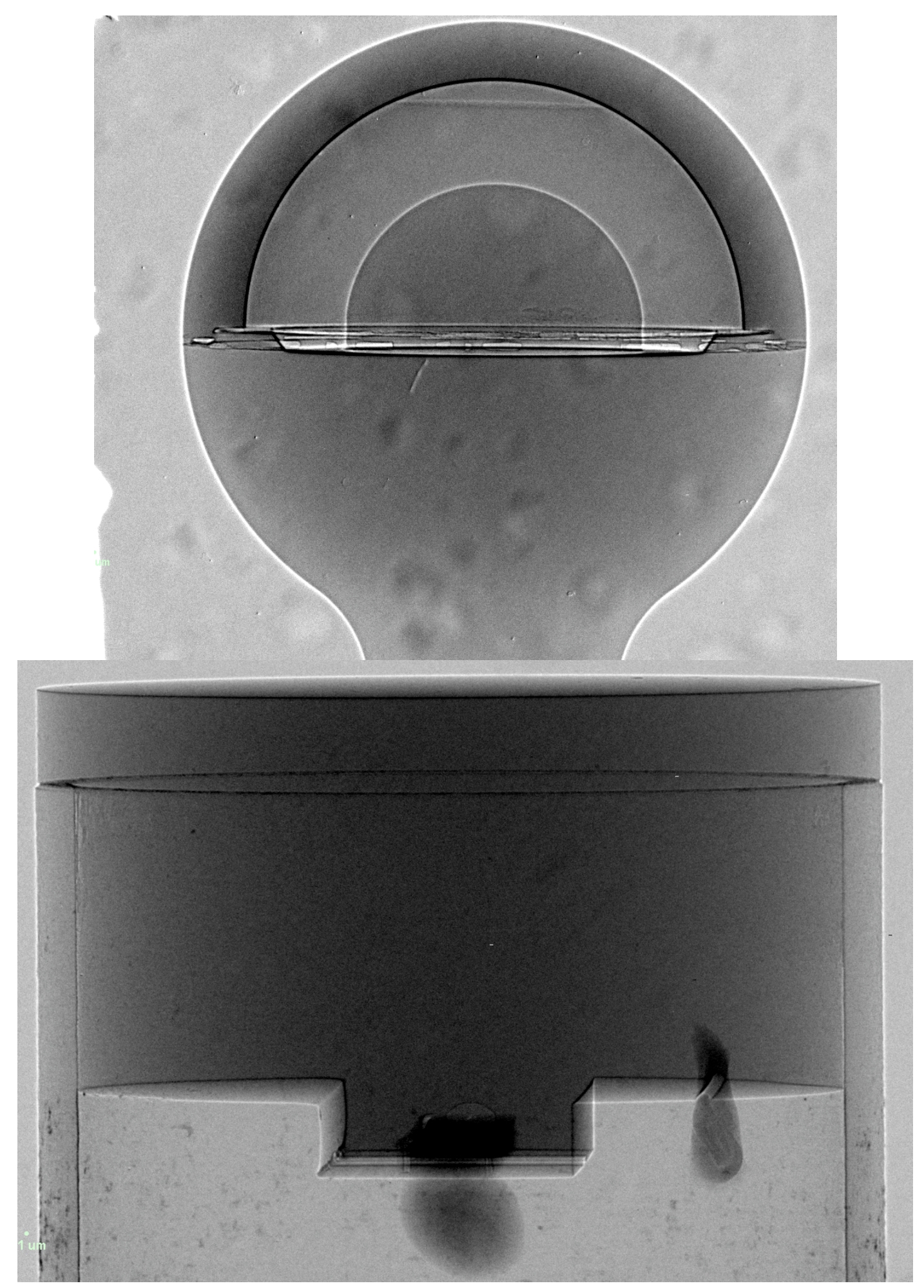

Figure 5. Percent transmission images of the spherical (top) and cylindrical (bottom) reference standards acquired with the Xradia $\mu$ XCT system. In these images, darker regions may indicate longer path lengths, or greater material density, while light areas may indicate shorter path lengths, or less density of material. Note: the two large dark areas on the cylindrical standard (bottom) are fiducial paint markers. Note: the soft blemishes seen in the images are believed to arise from the scintillator. 
The higher resolution modes were utilized to image small portions of each reference standard and are shown in Figure 6 below. The field of view of the system was not large enough to acquire full $\mathrm{CT}$ data sets at this higher resolution mode.
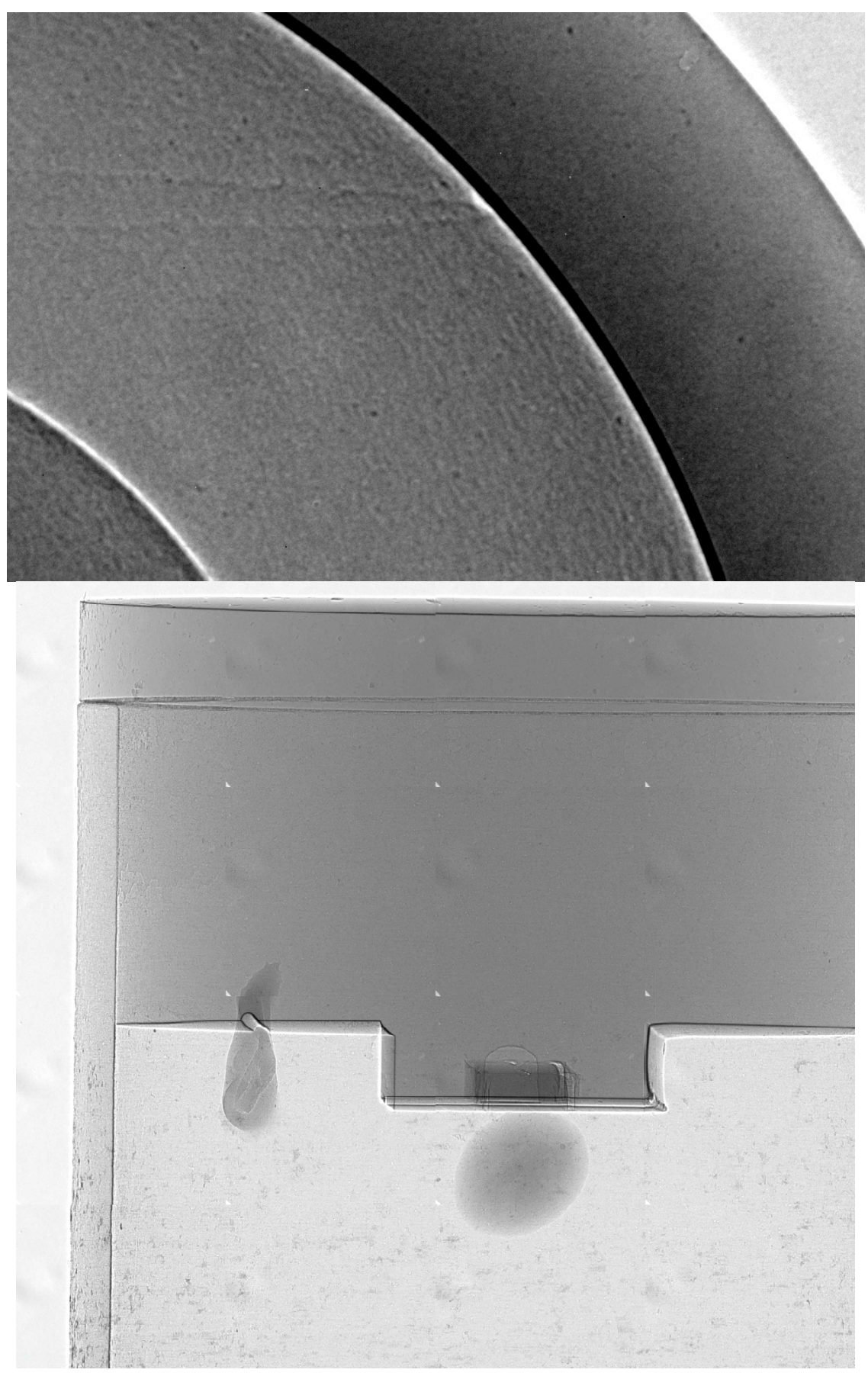

Figure 6. Top: High resolution mode image $(0.47 \mu \mathrm{m}$ pixels size at object) of the upper right quadrant of the spherical reference standard. Note: the $10-\mu \mathrm{m}$ radius groove in the aerogel can be seen in this image. Bottom: High resolution mode tiled mosaic image $(0.5 \mu \mathrm{m}$ pixel size at object) of one side of the cylindrical reference standard. Note the dustlike particles on the outside of the Be tube. 


\section{CT results of reference standards}

For CT data acquisition projection images were acquired over a minimum $180^{\circ}$ angular range for both standards. For the spherical standard, two CT scans were performed, each consisting of 721 projection images over $180^{\circ}\left(-90^{\circ}\right.$ to $+90^{\circ}$, with $0.25^{\circ}$ steps $)$. For the first scan, the exposure time was $8 \mathrm{sec}$ per image for a total acquisition time of $84 \mathrm{~min}$. A longer scan was then performed, using 80 seconds per projection image for a total acquisition time of approximately 16 hours. For the cylindrical standard 751 projections were acquired from $-93^{\circ}$ to $+93^{\circ}$, with $0.25^{\circ}$ steps. The cylindrical reference standard was scanned for 100 seconds per projection for a total of approximately 21 hours. Table 3 summarizes some of the CT scan parameters used for the spherical and cylindrical reference standards.

Table 3. Summary of CT data acquisition parameters used to scan the reference standards.

\begin{tabular}{|c|c|c|c|c|}
\hline $\begin{array}{c}\text { Reference } \\
\text { Standard }\end{array}$ & $\begin{array}{c}\text { Pixel size at } \\
\text { object }(\boldsymbol{\mu m})\end{array}$ & $\begin{array}{c}\text { Total number } \\
\text { of projections }\end{array}$ & $\begin{array}{c}\text { Time per } \\
\text { projection }(\mathbf{s})\end{array}$ & $\begin{array}{c}\text { Total scan } \\
\text { time }(\mathbf{h r s})\end{array}$ \\
\hline Spherical & 1.45 & 721 & 8 & $\sim 2$ \\
\hline Cylindrical & 2.31 & 751 & 80 & $\sim 16$ \\
\hline
\end{tabular}

From the transmission images (shown in Figure 5), attenuation radiographs were created $\left[\ln \left(\mathrm{I}_{0} / \mathrm{I}\right)\right]$. Detector imbalances were then minimized to avoid ring artifacts in the reconstructed images. Each set of attenuation radiographs was then reconstructed into a $\mathrm{CT}$ volume using a proprietary reconstruction algorithm based on the standard Filtered Back Projection (FBP) algorithm. The resulting CT volumes may then be sliced along any designated orthogonal axes for analysis.

Many important features within the reference standards are clearly seen in the CT images. In the spherical reference standard, for example, there appear to be several anomalies at or near the step-joint. As seen in Figure 5 and shown in Figure 7 below, excess material, most likely glue that has wicked into the aerogel, can be easily seen inside the step-joint. Voids in the joint itself can also be easily seen. In Figure 7-bottom, the air gap at the top edge of the aerogel is clearly visible, and the left side of the step joint does not look perfectly joined. Upon qualitative inspection of Figure 7 and additional CT slices, the inner hemisphere, meant to represent the double shell target, does not appear perfectly round. Reconstructed CT images from the cylindrical reference standard are shown in Figure 8. 


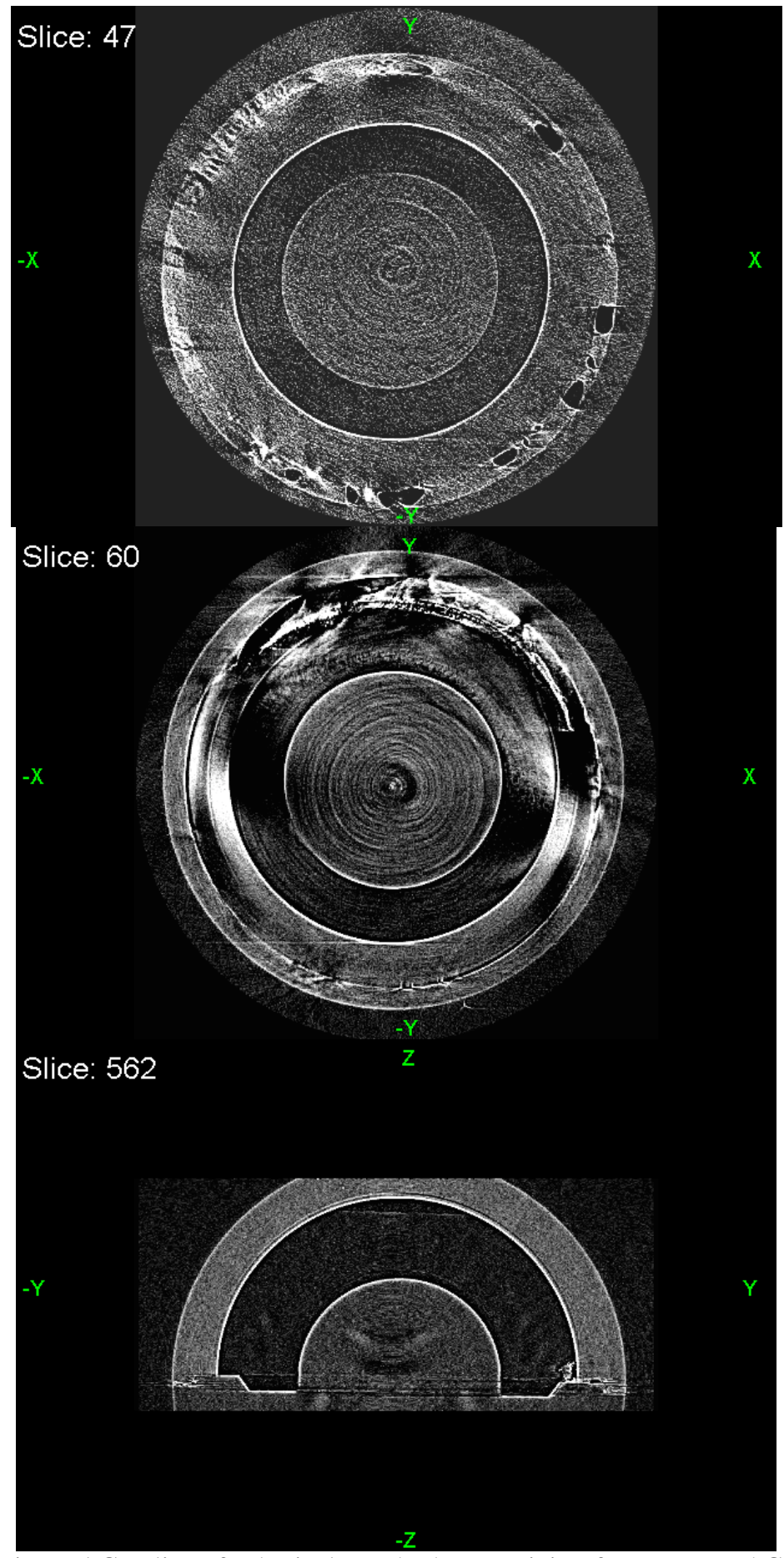

Figure 7. Top: Horizontal CT slice of spherical standard at step joint, from 7 second CT data acquisition. Middle: Horizontal CT slice of spherical standard at step joint, from 80 second CT data acquisition. Note: both CT slices show region of unbonded glue in the step joint. Bottom: 80 second CT slice through the vertical axis. Note: excess glue can be side on the right side of the step joint. The top and bottom surfaces of the aerogel can also be seen. 


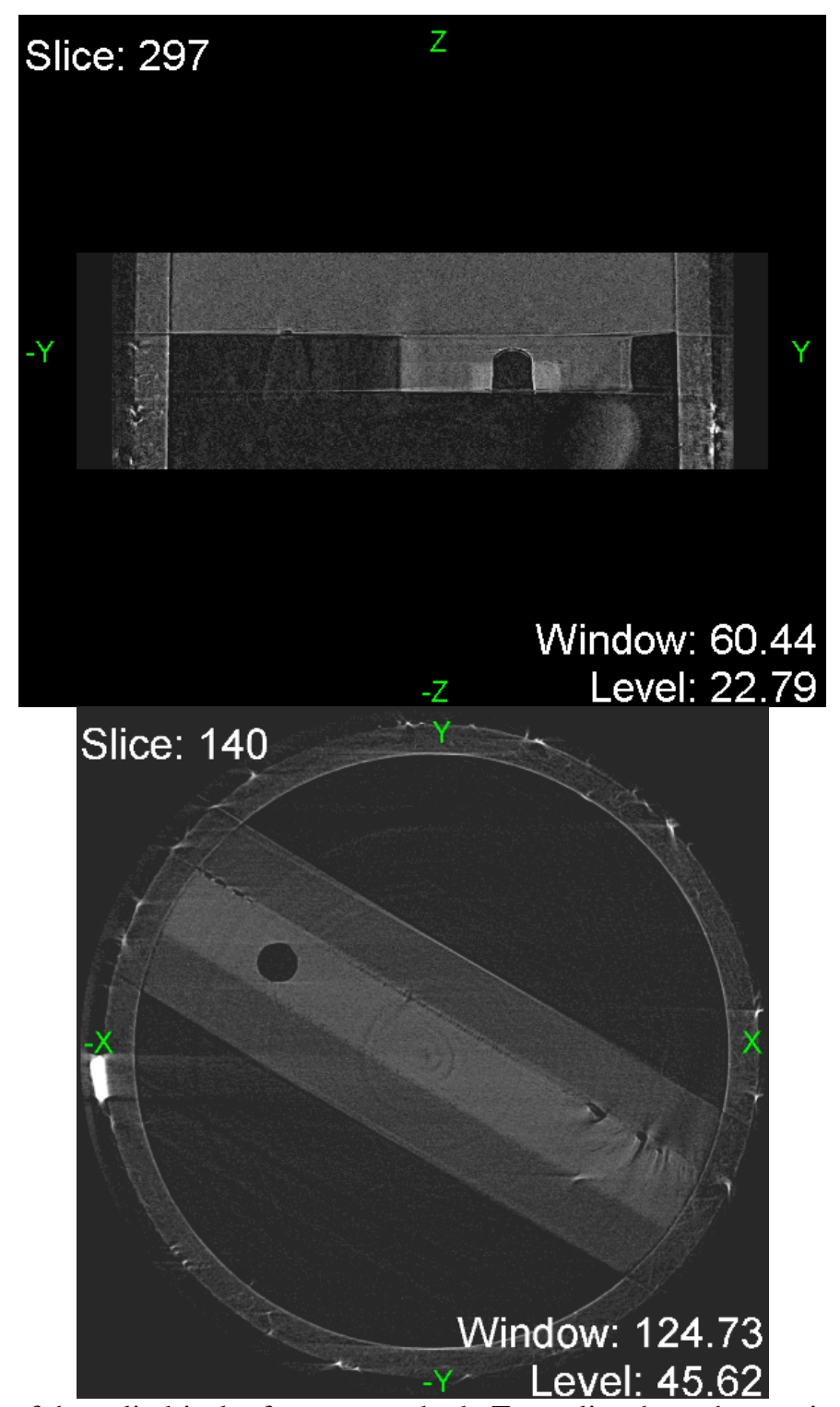

Figure 8. CT images of the cylindrical reference standard. Top: slice through a vertical plane. Bottom: slice through a horizontal plane. Note in both images the highly attenuating dustlike particles on the outside of the Be tube.

Much work needs to be done to quantify the CT data and qualitative observations described here. Any quantified data resulting from this project must be relevant to the target design and fabrication communities. To this end we are working with those groups to identify data of interest. The information that has been determined to be of interest for the spherical reference standard include: quantifying the $2-\mu \mathrm{m}$ gap built into the outer half of the step joint in the spherical reference standard; determining if the inner step joint is completely joined; qualitatively identifying flaws such as voids, and the wicking of glue; determining distributions of identified flaws, including maximum volumes, total number, and volume fractions; measuring to $1 \mu \mathrm{m}$ the concentricity of the outer hemishell and inner hemisphere of the spherical standard; quantifying wall thicknesses, including the mean and standard deviations; measuring the volume of the air gap between 
the aerogel and the $\mathrm{BrCH}$ upper hemisphere; and measuring the $10-\mu \mathrm{m}$ radius groove in the aerogel. Data of interest to be measured for the cylindrical reference standard include: measuring the thickness of the interface between the slot and the surface; measuring uniformity and voids within the bond; and measuring the interface between the foam and the polystyrene.

In addition, we are working to design and build additional objects that can be used to further quantify DR and CT system performance. A new edge has been designed and manufactured to more suitably measure the edge response function and subsequently the MTF for radiographs for the different DR/CT systems. We are also investigating ways to calculate the MTFs for CT images from existing data. To support this effort, a series of CT MTF phantoms have been fabricated from tubes of different material.

\section{As-built models from CT data}

An attempt was made to segment the Xradia spherical target data set for the purpose of building a model. This "as-built modeling" procedure is documented in [Waters, et al. 2004]. Unfortunately, the only Xradia CT images available to us at the time were those that had been deconvolved with the point spread function (psf). As discussed earlier in this report, these deconvolved images yield high contrast edges. However, attenuation values in the material are lost in the deconvolution process. Segmentation by thresholding is not possible using psf-deconvolved images. Unprocessed CT images are required for segmentation by thresholding.

\section{Summary}

We have begun to quantitatively measure Xradia's $\mu$ XCT digital radiography and computed tomography system performance. $\mu \mathrm{XCT}$ 's performance has been determined for mesoscale objects (reference standards of mm extent with $\mu \mathrm{m}$ features). CT data has been acquired for the spherical and cylindrical reference standards.

\section{Future work}

As mentioned previously in this report, an improved edge has been designed and manufactured for use in measuring MTF of DR systems. We plan to acquire DR images of the new edge to measure DR MTF in the near future. In addition, we have acquired several tubes made of different materials relevant to HEDP experiments. We plan to acquire CT data for these tube objects and use the data to measure MTF for CT performance. The quantitative measurements we have and will acquire will then be used to compare performance characteristics between various systems, including synchrotron and other commercially available and internally-developed DR/CT systems. Finally, appropriate data mining tools and techniques must be determined and possibly developed to extract quantitative and useful information from the three-dimensional CT data sets.

\section{References}


Hasegawa, B., The Physics of Medical X-ray imaging, Madison, WI, Medical Physics Publishing, 1991.

Hibbard, R., M. Bono, A. Waters, and H. Martz, "TechBase Final Report for: Production and Documentation of Mesoscale Metrology and Characterization Reference Standards", Lawrence Livermore National Laboratory, Livermore, CA, UCRL-ID-XXXX (2003).

Logan, C., J. Haskins, K. Morales, E. Updike, J. Fugina, T. Lavietes, D. Schneberk, G. Schmid, K. Springer, P. Soltani, and K. Swartz, "Evaluation of an Amorphous Selenium Array for Industrial X-Ray Imaging", Lawrence Livermore National Laboratory, Livermore, CA, UCRL-ID-132315 (1998).

Logan, C., Y-M Wang, M. Aufderheide, and H. Martz, "X-Ray Energies Appropriate for Imaging NIF High Energy Density Capsules", Lawrence Livermore National Laboratory, Livermore, CA, UCRL-ID-145954 (2001).

Waters, A., H. Martz, C. Logan, J. Gross and D. Chinn, "KCAT Performance Summary - Update”, Lawrence Livermore National Laboratory, Livermore, CA, UCRL-TR-203519 (2004). 